\title{
ciência
plưral
}

\section{EXPECTATIVA E PRÁTICA MATERNA DO ALEITAMENTO EXCLUSIVO E A SAÚDE BUCAL DO BEBÊ}

\section{Expectation and Practice of Exclusive Maternal Breastfeeding and Oral Health Baby}

Suzely Adas Saliba Moimaz • Professora Titular do Departamento de Odontologia Infantil e Social da Faculdade de Odontologia de Araçatuba (UNESP) Araçatuba-SP, Brasil. E-mail: sasaliba@foa.unesp.br

Gleice Tibauje Vicente Ramirez • Graduanda do curso de Odontologia da Faculdade de Odontologia de Araçatuba (UNESP) Araçatuba-SP, Brasil. E-mail: ramirez.tvgleice@gmail.com

Tânia Adas Saliba - Professora Assistente Doutora do Departamento de Odontologia Infantil e Social da Faculdade de Odontologia de Araçatuba (UNESP) Araçatuba-SP, Brasil. E-mail: taniasaliba@foa.unesp.br

Orlando Saliba - Professor Titular do Departamento de Odontologia Infantil e Social da Faculdade de Odontologia de Araçatuba (UNESP) Araçatuba-SP, Brasil. E-mail: osaliba@foa.unesp.br

Cléa Adas Saliba Garbin - Professora Titular do Departamento de Odontologia Infantil e Social da Faculdade de Odontologia de Araçatuba (UNESP) Araçatuba-SP, Brasil. Faculdade de Odontologia, UNESP Universidade Estadual Paulista, Araçatuba, SP, Brasil. E-mail: cgarbin@foa.unesp.br

Autora responsável pela correspondência:

Suzely Adas Saliba Moimaz. Professora Titular do Departamento de Odontologia Infantil e Social da Faculdade de Odontologia de Araçatuba (UNESP) Araçatuba-SP, Brasil. E-mail: sasaliba@foa.unesp.br 


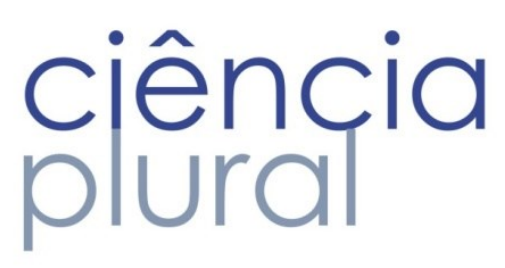

RESUMO

Introdução: $O$ aleitamento materno exclusivo (AME) é fundamental para a saúde do bebê, entretanto 0 sucesso da sua prática nem sempre é alcançado, pois depende de diferentes determinantes. Objetivos: Verificar a expectativa das gestantes em relação ao AME e percepção sobre a importância do aleitamento e a sua relação com a saúde bucal do filho e posteriormente acompanhar a prática durante os seis primeiros meses de vida do bebê para realização de inquérito e exame bucal da mãe e do filho. Métodos: Foi realizado um estudo longitudinal com 74 pares de mães e bebês $(n=148)$. No último trimestre de gestação, nas Unidades de Saúde da Família, as mulheres foram entrevistadas e visitadas nos domicílios, aos seis meses de idade do bebê. Resultados: Verificou-se que 95,95\% das entrevistadas pretendiam amamentar seu filho; $74 \%$ queriam AME até o sexto mês de idade do bebê, entretanto, após seis meses, $63,51 \%$ das mães estavam amamentando e apenas 18\% estavam em AME; $31,05 \%$ das mães tiveram dificuldades para amamentar. Com relação à saúde bucal $98,65 \%$ das gestantes pretendiam limpar a boca do bebê e levá-lo ao cirurgiãodentista; 36,49\% levariam chupeta ao hospital e 29,73\% achavam que amamentar não era importante para a saúde bucal do bebê. Após seis meses do nascimento do bebê; $63,51 \%$ limpavam a boca do bebê; $91,89 \%$ ainda não haviam levado seus bebês ao cirurgião-dentista e $25,68 \%$ levaram chupeta ao hospital. Em relação a saúde bucal, $32,72 \%$ das mães possuíam mancha branca. Do total de 74 bebês, 16 possuíam dentes aos seis meses de idade, três apresentavam manchas brancas e não estavam em AME. Conclusão: A expectativa de AME não se configurou totalmente na prática. A taxa de AME aos seis meses foi baixa, portanto a identificação das barreiras é importante para o estabelecimento de estratégias de promoção de saúde para superá-las.

Palavras-chave: Aleitamento Materno, Prática, Saúde Bucal.

\section{ABSTRACT}

Introduction: The exclusive breastfeeding (EBF) is fundamental for the health of child, however the sucess of the practice isn't always accomplished, because it depends on different determinants. Objectives: Verify the expectations of pregnant women in relation to exclusive breastfeeding and the importance of breastfeeding and its relationship with the oral health of the child and then accompany the practice during the first six months of the baby's life for making inquiries and oral examination of the mother and baby. Methods: It was longitudinal study with 74 pairs of mothers and children $(n=148)$. In the last trimester of pregnancy, in the Family Heath Units, the women were interviewed and visited in the homes, at six months old baby. Results: It was found that $95.95 \%$ of respondents wanted to breastfeed her child; $74 \%$ wanted EBF up to six months old baby, however, after six months, $63.51 \%$ of mothers were breastfeeding and only $18 \%$ were on EBF; $31.05 \%$ of the mothers had trouble breastfeeding. Regarding oral health $98.65 \%$ of the women wanted to clean the baby's mouth and take him to the dentist; $36.49 \%$ lead pacifier to the hospital and $29.73 \%$ felt that breastfeeding was not important to the oral health of the baby. After sixmonths of the baby's birth; $63.51 \%$ cleared the baby's mouth; $91.89 \%$ had not yet taken their babies to the dentist and $25.68 \%$ led pacifier to the hospital. Regarding oral health, $32.72 \%$ of the mothers had a white spot. Of the total of 74 babies, 16 had teeth at six months of age, three had white spots and were not in EBF. Conclusion: The expectation of exclusive breastfeeding is not has configured in practice and that maternal knowledge relating to oral health of children with breastfeeding is low. The exclusive breastfeeding rate at six months was low, so the identification of barriers is important for the establishment of health promotion strategies to overcome them.

Key words: Breastfeeding, Pregnant women, Oral health. 


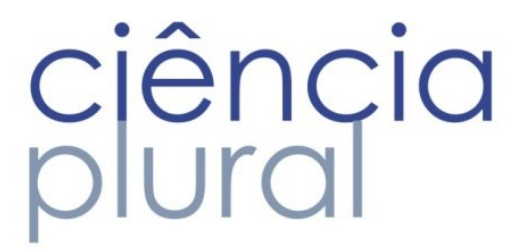

Introdução

O aleitamento materno exclusivo (AME) é fundamental para o crescimento, desenvolvimento, saúde e nutrição dos bebês, sendo uma intervenção simples e efetiva ${ }^{1,2}$. Estudos indicam que o AME aos seis meses de idade, diminui os riscos de hospitalização na infância para uma série de doenças infantis comuns entre crianças no primeiro ano de vida ${ }^{3}$, além de promover um intenso trabalho da musculatura peribucal, influênciando o desenvolvimento correto dos padrões ósseos e musculares, pois o crescimento facial harmônico é fundamental não apenas por questões estéticas, mas também para o bom funcionamento do sistema mastigatório, evitando a futura necessidade de correção ortodôntica, protética e até mesmo cirúrgica do conjunto dento-maxilo-mandibular e suas estruturas adjacentes, além de gerar fadiga nesses músculos, fazendo com que a criança satisfaça seu instinto de sugar e não necessite de uma sucção não-nutritiva, evitando o uso de bicos artificiais ${ }^{2,4}$.

O progresso do aumento das taxas do aleitamento materno (AM) no mundo iniciou-se a partir da Declaração de Innocenti em 1990, que abordava a promoção, proteção e apoio ao aleitamento materno, e trouxe melhoria para a criança e práticas de alimentação infantil em todo o mundo, inclusive no Brasil 5 . Porém mesmo com o aumento das taxas do AM, estas ainda estão bem aquém das taxas recomendadas pelos órgãos nacionais e internacionais de saúde, isto é, de aleitamento materno exclusivo (AME) até os seis meses de idade do bebê e AM complementar até os dois anos de idade ou mais ${ }^{6}$. Evidências recentes também indicam que aproximadamente $50 \%$ das crianças são amamentadas exclusivamente em apenas 28 países 7 .

No Brasil foi verificado que apenas $41 \%$ das crianças menores de seis meses de idade foram amamentadas exclusivamente no peito. Um estudo realizado nas capitais brasileiras e Distrito Federal demonstrou que a duração mediana do AME foi de 54,1 dias (1,8 meses) e a duração mediana do AM de 341,6 dias (11,2 meses). Constatou-se aumento da prevalência de AME em menores de quatro meses no conjunto das capitais brasileiras e DF, de 35,5\%, em 1999, para 51,2\%, em 2008. Apesar das taxas de AME e AM terem aumentado no Brasil nos últimos anos, ainda se encontram aquém da taxa ideal 8 .

O sucesso do aleitamento materno depende de muitos determinantes, dentre eles: apoio familiar e do cônjuge, nível sócio econômico, grau de escolaridade da mãe, idade da mãe, trabalho materno, urbanização, condições do parto, bem como a intenção da mãe de amamentar e experiência anterior à essa ${ }^{9}$. Existem também problemas fisiológicos ao ato de amamentar que dificultam sua prática: mastites, ingurgitamento mamário, fissuras nos mamilos, insuficiência quantitativa de leite, entre outros ${ }^{10}$.

Considerando as baixas taxas de AME e a complexidade envolvida no processo de saúde e mudança 


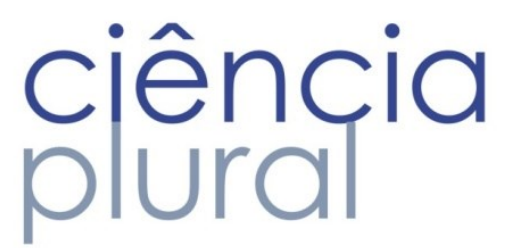

comportamental, a multiplicidade de fatores envolvidos na prática do AME tornam importante 0 desenvolvimento de pesquisa sobre o tema. Poucos são os estudos publicados na literatura com seguimentos de gestantes ou pares de mães e bebês. $O$ conhecimento das dificuldades enfrentadas na prática diária do AME pode levar ao estabelecimento de estratégias mais eficazes para sua prevalência.Nesse sentido, 0 presente estudo objetivou verificar a expectativa de gestantes em relação ao aleitamento materno exclusivo; a percepção sobre a importância do aleitamento e a sua relação com a saúde bucal do filho e posteriormente acompanhar a prática do AME, durante os seis primeiros meses de vida do bebê para realização de inquérito e exame bucal da mãe e do filho.

\section{Materiais e Métodos}

Foi realizado um treinamento técnico para entrevistador e anotador da coleta de dados do estudo durante duas semanas sobre: esclarecimento quanto aos objetivos do projeto de pesquisa, iniciação à pesquisa científica, métodos da aplicação do formulário e preenchimento do instrumento da coleta de dados, conceitos teóricos sobre aleitamento materno e alimentação infantil , a fim de padronizar os procedimentos na coleta de dados e orientar as visitas domiciliares. Também foi feito um estudo piloto realizado com $10 \%$ da amostra, visando diminuir erros, manter a uniformidade das informações e testar o instrumento de coleta de dados.

Como critérios de inclusão das participantes da pesquisa, foram consideradas: gestantes que estavam no último trimestre de gestação, que não tinham nenhuma doença sistêmica que impossibilitasse a prática do aleitamento materno, que não tinham problemas na mama que dificultasse a amamentação, mães que não tinham mais de um filho (gêmeos, trigêmeos, etc.) e as gestantes que consentissem em participar do estudo.

Como critérios de exclusão foram considerados: mães ou bebês com algum tipo de doença, que impossibilitasse a prática do aleitamento materno (HIV, problemas congênitos, entre outros), morte do bebê e prematuros (nascidos com menos de 37 semanas).

Realizou - se um estudo longitudinal, com 100 mulheres desde a gestação até os seis meses de idade de seu filho, todavia houve uma perda no número da amostra de $26 \%$ durante a fase de acompanhamento, aos seis meses de idade das crianças, em sua maioria por mudança de endereço e número telefônico e, em alguns casos, bebês prematuros nascidos com menos de 37 semanas, diminuindo o número para 74 mulheres.

No último trimestre de gestação, as gestantes que aceitaram participar da pesquisa, assinando o 


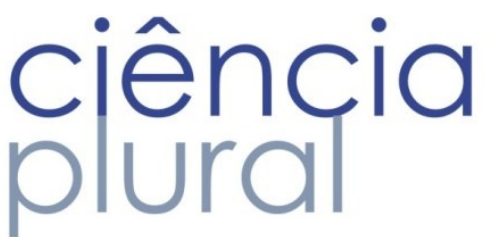

consentimento de livre e esclarecido, após registro de dados cadastrais, foram entrevistadas sobre a intenção e expectativa em amamentar e saúde bucal do bebê, durante horário de atendimento do médico obstetra em Unidades de Saúde da Família de um município do estado de São Paulo, Brasil. Com o término da entrevista, todas as gestantes participaram de atividades educativas, assistiram a um vídeo e receberam panfletos sobre o incentivo da prática de aleitamento materno e instalação de hábitos saudáveis, desenvolvido com base nas recomendações da Organização Mundial de Saúde em associação com a UNICEF ${ }^{11,12 .}$

Mediante a um calendário estabelecido nos dados da data provável do parto, as 74 mulheres foram visitadas em domicílio quando seus filhos completaram seis meses de idade, e entrevistadas, foram indagadas sobre a prática do aleitamento materno e saúde bucal do bebê; sendo registrada dessa maneira, variáveis sobre percepção materna da saúde bucal do bebê e do aleitamento1. Embora tenham sido explanado os objetivos da pesquisa, e tendo sido assinado o consentimento livre esclarecido, apenas 55 participantes autorizaram realizar o exame bucal.

Os dados recolhidos foram digitados e analisados no Programa Epi Info 7, para levantamentos epidemiológicos. A pesquisa em questão foi aprovada pelo comitê de Ética em Pesquisa Humana pela Faculdade de Odontologia - Júlio de Mesquista Filho, Araçatuba - SP, pelo parecer 904.717.

\section{Resultados}

As mães apresentaram em média 25,81 anos de idade, variando entre 17 e 37 anos e renda familiar de 1.81 salários mínimos. Havia 4,24\% pardas; $43,24 \%$ brancas e $13,52 \%$ negras. Do total, $50 \%$ eram amasiadas; $33,8 \%$ casadas e $16,2 \%$ solteiras. A escolaridade configurou-se como baixa, de acordo com os dados observados: $15 \%$ apresentaram ensino fundamental incompleto, $18 \%$ fundamental completo, $24 \%$ médio incompleto, $38 \%$ médio completo e $05 \%$ superior incompleto.

Quadro 1: Distribuição numérica e percentual das mães, em relação à expectativa e prática do aleitamento e saúde bucal do bebê. Araçatuba-SP, Brasil, 2016.

\begin{tabular}{|c|cc|cc|cc|}
\hline \multirow{2}{*}{ Questão } & \multicolumn{2}{|c|}{ Expectativa } & \multicolumn{2}{|c|}{ Prática } & \multicolumn{2}{c|}{ Total } \\
\cline { 2 - 8 } & $\mathrm{n}$ & $\%$ & $\mathrm{n}$ & $\%$ & $\mathrm{n}$ & $\%$ \\
\hline Amamentar o filho no peito & 71 & 95,95 & 47 & 63,51 & 74 & 100,0 \\
\hline AME até os seis meses & 55 & 74,00 & 13 & 18,00 & 74 & 100,0 \\
\hline Limpar a boca do bebê & 73 & 98,65 & 47 & 63,51 & 74 & 100,0 \\
\hline Levar seu filho ao cirurgião - dentista & 73 & 98,65 & 06 & 08,11 & 74 & 100,0 \\
\hline Levar chupeta ao hospital & 27 & 36,49 & 19 & 25,68 & 74 & 100,0 \\
\hline
\end{tabular}




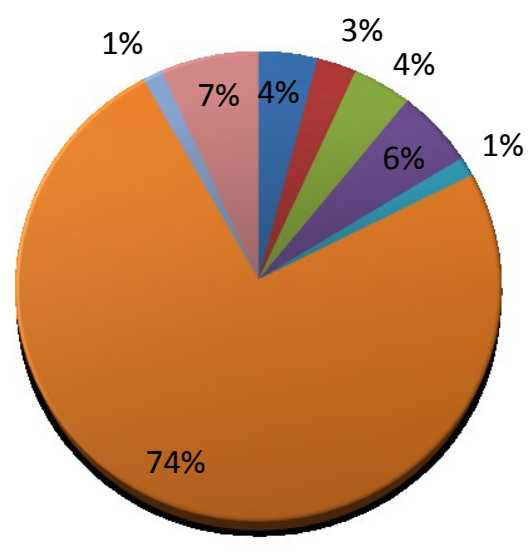

$\square 1$ mês

$\square 2$ meses

$\square 3$ meses

$\square 4$ meses

$\square 5$ meses

$\square 6$ meses

$\square 7$ meses

$\square$ Não sabe

Figura 1: Distribuição percentual, segundo respostas das gestantes sobre a expectativa materna em relação ao tempo de aleitamento materno exclusivo. Araçatuba-SP, Brasil, 2016.

Quadro 2: Distribuição numérica e percentual das mães, segundo percepção e comportamentos relacionados à saúde do bebê. Araçatuba-SP, Brasil, 2016.

\begin{tabular}{|c|cc|cc|cc|}
\hline Questão & \multicolumn{2}{|c|}{ Sim } & \multicolumn{2}{c|}{ Não } & \multicolumn{2}{c|}{ Total } \\
\cline { 2 - 7 } & $\mathbf{n}$ & $\%$ & $\mathbf{n}$ & $\%$ & $\mathbf{n}$ & $\%$ \\
\hline $\begin{array}{c}\text { Você acha que amamentar seu filho no peito traz } \\
\text { benefícios para a saúde bucal do bebê? }\end{array}$ & 69 & 93,24 & 05 & 06,76 & 74 & 100,0 \\
\hline $\begin{array}{c}\text { Já percebeu alguma alteração na boca do seu } \\
\text { bebê? }\end{array}$ & 39 & 52,70 & 35 & 47,30 & 74 & 100,0 \\
\hline As escovas de dente, são compartilhadas? & 05 & 06,76 & 69 & 93,24 & 74 & 100,0 \\
\hline Teve dificuldades em amamentar & 23 & 31,08 & 51 & 68,92 & 74 & 100,0 \\
\hline
\end{tabular}




\section{ciência

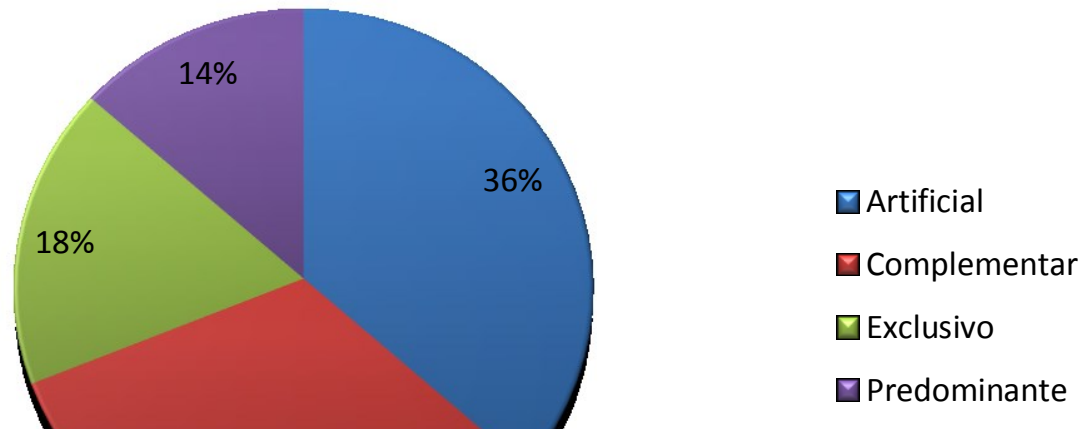

Figura 2: Distribuição percentual das mães, segundo a modalidade de aleitamento praticado aos seis meses de idade do bebê. Araçatuba-SP, Brasil, 2016.

Dentre os exames bucais realizados em 55 mães 32,72\% ( $n=18$ ) possuíam lesão branca ativa e 47,27\% $(n=26)$ presença de biofilme bacteriano. Mediante análise estatística realizada por meio do Teste do Quiquadrado $(p>0,05)$ entre a associação do nível de escolaridade e variáveis como lesão branca $(0,4325)$ e a presença de biofilme bacteriano dentário da mãe $(0,2583)$, não foi encontrada associação significativa para nenhumas das variáveis.

Quanto aos bebês, apenas 16 deles $(21,6 \%)$ possuíam dentes na boca aos seis meses de idade; $18,75 \%$ ( $n=3$ ) já possuíam lesão branca, destes, 33\% estavam sob aleitamento predominante e $67 \%$ sob aleitamento complementar.

\section{Discussão}

O ato de amamentar é de extrema importância para a mãe, promovendo melhor involução genital pósparto, diminui a incidência de câncer de mama e útero, previne a osteoporose, promove amenorreia pós-parto e retarda nova gestação. Para o bebê, o leite materno possui conteúdo nutricional, prevenindo alergias e problemas respiratórios, tem uma melhor absorção interna, auxilia na formação das defesas imunológicas, propicia desenvolvimento psicológico mais adequado, diminui o risco de hospitalização na infância para uma série de doenças próprias dessa fase da vida, e quando amamentado direto no peito, o bebê é estimulado para o correto desenvolvimento esquelético e muscular ${ }^{1-4,13}$. Entretanto, como visto neste estudo, algumas gestantes $(6,76 \%)$ desconhecem os benefícios do aleitamento para a saúde bucal, que é o desenvolvimento 


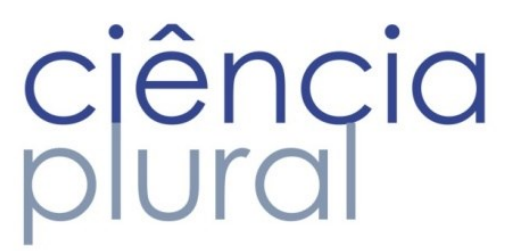

dento-facial e de favorecer a obtenção de uma oclusão dentária normal, prevenindo a síndrome da respiração bucal e a deglutição atípica9 ${ }^{9}$.

Com relação às taxas de $\mathrm{AME}$ e $\mathrm{AM}$, neste estudo, a prática ficou aquém da expectativa. Apesar da maioria das gestantes terem sido orientadas e possuírem conhecimento sobre o aleitamento materno, houve desmame precoce. A orientação sobre aleitamento e outros assuntos relacionados à saúde materno-infantil é importante, entretanto, o apoio e o acompanhamento das mães são fundamentais para que se tenha sucesso na prática da amamentação ${ }^{15,16}$.

Houve alta taxa de dificuldades em amamentar recém-nascidos identificadas pelas mães. Dos problemas relatados os mais frequentes são: "não tenho bico", "dificuldade da criança pegar o seio" e "ferida no bico do peito". Seria muito interessante que as mães tivessem fácil acesso a serviços de saúde aptos a auxiliá-las no período de amamentação, bem como fossem cada vez mais divulgadas, para a população em geral, conhecimentos sobre a técnica do aleitamento natural1,10,17.

A promoção do aleitamento materno deveria ser vista como ação prioritária para a melhoria da saúde e da qualidade de vida das crianças e de suas famílias. Promover o AM pode ser um bom exemplo de política pública que envolve a família, comunidade, governos e sociedade civil, com baixo custo e excelente impacto sobre o desenvolvimento e saúde infantili13, 18 .

Na prática da amamentação, um dos cuidados essenciais é não instituir à mulher toda a culpa por não conseguir amamentar, já que algumas vezes a mulher deseja amamentar, mas não encontra 0 apoio necessário, além de se deparar com dificuldades que para ela podem ser intransponíveis, de modo que a principal razão para a interrupção prematura da amamentação é a dificuldade em lidar com o aleitamento materno ${ }^{19}$. Por isso é importante o acompanhamento das lactantes por profissionais de saúde com objetivo de orientar e promover a prática do $\mathrm{AM}^{17}$. Com relação à saúde bucal, embora haja indícios, não há evidências suficientes que comprovem que o leite materno possa estar associado com o surgimento de cárie, sendo essa relação complexa e confundida por muitas variáveis ${ }^{20}$.

A Academia Americana de Odontopediatria, sugere que a gengiva e os dentes dos bebês devem ser limpos suavemente pelos pais, após a amamentação. Dessa forma, o cirurgião-dentista deve incentivar e apoiar práticas de saúde bucal e amamentação ideal entre seus pacientes²1.

Entre os fatores de risco para interrupção do AME e do AM destacam-se o uso precoce da chupeta, falta de acompanhamento ambulatorial em serviço público, idade da mãe e trabalho materno ${ }^{1,2}$. Além do uso de chupeta estar associada com desmame precoce, também está relacionada à prevalência de oclusopatias, 


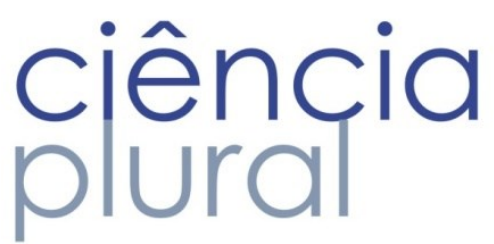

uma vez que crianças com hábitos de sucção não nutritivos durante a fase de dentição decídua apresentam elevada prevalência de má oclusão ${ }^{22}$. Ações de promoção de saúde, com foco nos fatores determinantes e condicionantes dessas anomalias craniofaciais, são essenciais para melhoria da qualidade de vida das crianças e suas famílias ${ }^{13}$. Portanto, o aleitamento materno, além de inúmeras vantagens, exerce papel preventivo na aquisição de hábitos de sucção não nutritivos e na prevalência de oclusopatias ${ }^{14}$.

Bebês que fazem uso de mamadeira no primeiro mês apresentam maior número de itens desfavoráveis tanto no posicionamento quanto na qualidade da pega, indicando técnica menos adequada; sugerindo que 0 uso de mamadeira influencia fortemente a técnica de amamentação 23 .

A intervenção e assistência materno - infantil pelos profissionais de saúde são importantes no incentivo do AM, apoiando e instruindo a nutriz, pelo acompanhamento pré-natal cuidadoso, formação de grupos de gestantes, alojamento conjunto, durante a puericultura e na promoção de campanha de incentivo ao aleitamento. Conhecendo os motivos que possam contribuir com o abandono da prática de aleitamento materno exclusivo, pode-se atuar melhor na inter-relação de fatores ambientais e socioculturais que influenciam na prática de amamentar, mesclando políticas públicas, benefícios, rotinas, ações de profissionais, apoio de pares etc., no sentido de prevenção desses fatores de forma mais direcionada e, portanto, mais eficaz. Além de conhecer os motivos que levam ao desmame precoce, é fundamental acompanhar e apoiar as mães para que as mesmas se sintam capazes de enfrentar as dificuldades que possam surgir24,25. É fundamental conhecer as percepções maternas sobre a importância do aleitamento materno e a saúde bucal de seus filhos, a fim de desenvolver ações de promoção, orientação, proteção e incentivo desta prática, devido aos inúmeros benefícios que traz para a saúde bucal e geral infantil. Nesse sentido, a odontologia preventiva busca promover estratégias de ação para a manutenção da saúde bucal e geral no âmbito familiar, assim como identificar e criar soluções para as dificuldades encontradas no dia-adia das famílias sendo de fundamental importância para que o AME ocorra até os seis meses de idade do bebê.

\section{Conclusões}

Conclui-se que a prática do aleitamento materno exclusivo ficou muito aquém da intenção inicial das mães. O sucesso do AME tem como determinante além da intenção materna em relação à prática, 0 acompanhamento deste público por profissionais de saúde, para identificação e resolução das barreiras observadas. 


\section{ciência plural}

\section{Agradecimentos}

Agradecemos ao pessoal do Departamento de Odontologia Infantil e Social que contribuíram para a realização desta pesquisa e ao Conselho Nacional de Desenvolvimento Científico e Tecnológico que apoiou financeiramente através do Programa Institucional de Bolsas de Iniciação Científica.

\section{Referências}

1. Rocha NB, Garbin AJI, Garbin CAS, Saliba O, Moimaz SAS. Estudo longitudinal sobre a prática de aleitamento materno e fatores associados ao desmame precoce. Pesquisa Brasileira em Odontopediatria e Clínica Integrada2013;13(4):337-342.

2. Rocha NB, Garbin AJI,Garbin CAS, Moimaz SAS, Saliba O, Gonçalves PE. Amamantamiento y hábitos de succión no nutritivos: un estudio de cohorte. Acta Odontol Venez. 2013;51(3):1-7.

3. Ajetunmobi OM, Whyte B, Chalmers J, Tappin DM, Wolfson L, Fleming M, et al. Breastfeeding Project Steering Group. Breastfeeding is associated with reduced childhood hospitalization: evidence from a Scottish Birth Cohort (1997-2009).J Pediatr. 2015 Mar;166(3):620-5. PMid: 25556021.

4. Ribeiro NME, Ribeiro MAS. Aleitamento materno e cárie do lactente e do pré-escolar: uma revisão crítica.J Pediatr. 2004;80(5 Suppl): S199-210.

5. World Health Organization. Regional Office for Europe. Comparative analysis of implementation of the Innocenti Declaration in WHO European Member States. Geneva: WHO;1999.

6. World Health Organization. Global strategy for infant and youngchild feeding. Geneva: WHO; 2003.

7. Unicef.State of the World's Children 2008. Child Survival. Disponivel em: https://www.unicef.org/publications/index_42623.html

8. Brasil. Ministério da Saúde. II Pesquisa de prevalência de aleitamento materno nas capitais brasileiras e distrito federal. Brasilia: Ministério da Saúde; 2009.

9. Araújo OD, Cunha AL, Lustosa LR, Nery IS, Mendonça RCM,Campelo SMA. Aleitamento materno: fatores que levam ao desmame precoce. Rev Bras Enferm. 2008 Jul-Aug;61(4):488-92.

10. Santos VLFD, Soler ZASG, Azoubel R. Alimentação de criançasno primeiro semestre de vida: enfoque no aleitamento materno exclusivo. Rev Bras Saúde Matern Infant. 2005 jul-set;5(3):283-91. 


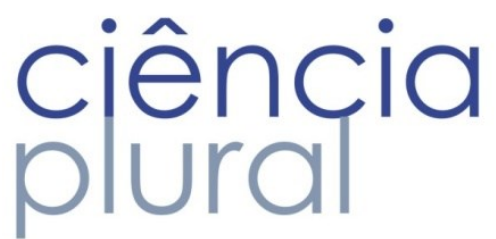

11. World Health Organization. The optimal duration of exclusive breastfeeding: Report of an Expert Consultation, Geneva, Switzerland. Geneva: WHO; 2001.

12. Unicef. The Breastfeeding Iniatives Exchange. Geneva: Unicef; 2002. Disponível em: https://www.unicef.org/programme/breastfeeding/baby

13. Moimaz SAS, Ros DT, Garbin CAS, Saliba NA. Aleitamento materno: benefícios à saúde e implicações na saúde bucal. Rev Paul Odontol. 2014;36(4):24-31.

14. Moimaz SAS, Rocha NB, Garbin AJI, Saliba O. A influência da prática do aleitamento materno na aquisição de hábitos de sucção não nutritivos e prevenção de oclusopatias. Rev Odontol UNESP. 2013 JanFeb;42(1):31-6.

15. Vannuchi MTO, Thomson Z, Escuder MML, Tacla MTGM, Vezozzo KMK, Castro LMCP, et al. Perfil do aleitamento materno em menores de um ano no Município de Londrina, Paraná. Rev Bras Saúde Matern Infant. 2005 Abr-Jun;5(2):155-62.

16. Moimaz SAS, Saliba O, Borges HC, Rocha NB, Saliba NA. Desmame Precoce: Falta de Conhecimento ou de Acompanhamento? Pesqui Bras Odontopediatr Clín Integr. 2013;13(1):53-9.

17. Moura EFA. Duração do período de aleitamento materno de crianças atendidas em ambulatório de pediatria. J Pediatr. 1997 Mar-Abr;73(2):106-10.

18. Parada CMGL, Carvalhaes MABL, Winckler CC, Winckler LA, Winckler VC. Situação do aleitamento materno em população assistida pelo programa de saúde da família-PSF. Rev Latino-Am Enfermagem. 2005 Maio-Jun;13(3):407-14.

19. Hill PD. The enigma of insufficient milk supply. MCN Am J Matern Child Nurs. 1991 NovDec;16(6):312-6. PMid: 1824557.

20. Losso EM, Tavares MCR, SilvaJYB, Urban CAJ. Cárie precoce e severa na infância: uma abordagem integral.J Pediatr. 2009;85(4):295-300.

21. Salone LR, Vann WF Jr, Dee DL. Breastfeeding: an overview of oral and general health benefits. J Am Dent Assoc. 2013 Feb;144(2):143-51. PMid: 23372130. https://doi.org/10.14219/jada.archive.2013.0093.

22. Boeck EM, Pizzol KEDC, Barbosa EGP, Pires NCl, Lunardi N. Prevalência de má oclusão em crianças de 3 a 6 anos portadoras de hábito de sucção de dedo e/ou chupeta. Rev Odontol UNESP. 2013;42(2):1106. 


\section{ciência plural}

23. França MCT, Giuglianill ERJ, Oliveira LD, Weigertl EML, Espirito Santo LC, Köhler CV, et al. Uso de mamadeira no primeiro mês de vida: determinantes e influência na técnica de amamentação. Rev Saúde Pública 2008 Aug;42(4):607-14.

24. Chaves RG, Lamounier JA, César CC. Fatores associados com a duração do aleitamento materno. J Pediatr. 2007 May-Jun;83(3):241-6.

25. Toma TS, Rea MF. Benefícios da amamentação para a saúde da mulher e da criança: um ensaio sobre as evidências. Cad Saúde Pública. 2008;24(Supp 2):S235-46. 